former child. Severe attack of vaginitis, with much purulent discharge in which gonococci were found. Cultures showed cocci and few colonies of diplococci. Two weeks later gonococci were still found in the pus. A culture at this time showed only colonies of diplococci.

Case III. The following history was taken from the records by kind permission of Dr. Burrell : Child, one year and eight months old. Had been to Wellesley convalescing from an empyema, and returned to Out-Patient Department April 6th. Redness and slight ulceration was noted on labia minora and majora. Some discharge from vagina. April 8th, ulceration had spread rapidly. Labia majora red and slightly swollen. On retracting the labia a superficial ulcerative process of considerable extent was seen. The upper third of both labia minora had been destroyed, and the ulcerative process extended up on the left labium majus. The surface of the ulcer was grayish and the edges not indurated. April 10th, ulceration seemed to be extending but little, and the opposing surfaces of the labia minora were ulcerated. April 15th, ulceration slowly getting into better condition. General condition improving and child cried less. April 25th, ulcerations much diminished, and continued to improve till May 8th, when they were entirely healed.

At one time there was a fine papular eruption on the buttocks, which was of a very few days' duration only, and considered to be due to irritation. At entrance there was a temperature of $101.5^{\circ}$, which gradually fell to normal by the end of the first week. During the whole attack there was considerable purulent discharge, and this continued in a small amount after the ulceration had healed. The process, whatever its nature, yielded with great difficulty to antiseptic treatment. No gonococci could be found in the discharge. but a bacillus of varying length was found both within and outside the leucocytes. No other organism was present. Cultures were made and many bluish-white colonies of a short, thick bacillus with rounded ends developed in pure culture. This was transferred to other media; and its growth, gas production, acid formation (as seen on litmus agar and in milk), indol reaction and failure to stain by Gram's method, showed it to be identical with the bacillus coli communis.

Apropos of this last case is a urethritis in a male reported by Van-der Pluym and Laag. ${ }^{2}$ In this case a purulent discharge similar to gonorrhea developed seven days after coitus. There was also considerable febrile disturbauce. No gonococci could be found in the pus, but instead a bacillus of varying length was found within the leucocytes, from two to six or seven in a cell. Rarely was ove found free in the secretion.

Cultures were made, and the bacillus proved to be identical with the bacillus coli communis in all respects. The authors claim that this organism was the cause of the attack, because it was an acute infectious disease with febrile reaction. The bacillus was found within the cells, and all other bacteria were absent. Ibe prostitute from whom the disease was contracted could not be found to be examined. In this connection it will be remembered that Case XVII of my series of urethrites also showed the bacillus coli communis.

(To be contrnued.)

2 Centralbl. f. Bact., May, 1895.

\section{RECURRENT OCULO-MOTOR PALSY WITH A} CASE.1

BY G. E. DE SChWEINITZ, M.D., PHiladelphia.

RECURRENT oculo-motor palsy is of sufficient rarity and interest to justify the record of each additional case, and therefore I report the following example of this affection :

Kate Y., unmarried, aged thirty, American born, a cigarmaker by trade, applied to the Ophthalmic Department of the Jefferson Medical College in the latter part of December, 1894, for relief from violent attacks of neuralgia and complete oculo-motor palsy of the right side.

Family History. - Her mother suffered from right hemiplegia at the age of forty-one, never fully recovered, and died suddenly three years later. Two brothers and one sister are living and in good health. One brother died in infancy from an accident. The history on the paternal side of the family was not obtainable.

Personal History. - When one and one-half years of age, the patient became violently ill with the following symptoms: vomiting, convulsions, right divergent strabismus and ptosis. At the end of six weeks the ptosis disappeared, and the eye returned to its proper position. Since this date she has suffered from frequent attacks of neuralgia, almost always associated with divergence of the right eye and closure of the corresponding lid; occasionally, although the pain was severe, the ocular symptoms were absent.

During childhood the explosions of pain were frequent, occurring as often as three times a week ; lately the maximum number of attacks has been five or six a year, and sometimes she has been free from them for six months at a time.

With the exception of whooping-cough, measles, scarlatina and epidemic influenza (three years ago), each disease being accompanied by a violent attack of migraine and recurrence of the ocular palsy, she has enjoyed good health. Menstruation was established at thirteen years of age, is regular in appearance, and unaccompanied by pain. A history of syphilis was not obtained.

When she was about four years of age (although she is somewhat uncertain as to the date), the divergence, which had before that time disappeared pari passu with the subsidence of the ptosis, became more or less permanent, although she thinks that in her best condition, even though the eye turned outward, power still remained to bring it to the median line. In September, 1898, an unusually severe attack of neuralgia occurred, accompanied, as usual, by ptosis; but when the pain disappeared, the ptosis re. mained. Since the date of permanent ptosis she has suffered about half-a-dozen mild attacks of neuralgia, unaccompanied by notable ocular changes.

The Neuralgic Attack.- Preceded by dizziness, a full feeling of the head, swelling of the peri-orbital tissues and distention of the veins; the pain begins in the right eye and travels around the right side of the head to the occiput, where it finally settles and remains until the subsidence of the nerve-storm. Immediately following the onset of the pain, there is vomiting, which lasts from twelve to twenty-four hours, and leaves the patient utterly dejected and exhausted.

When examined (December, 1894), the following conditions were present. Complete paralysis of the oculomotor nerve, the divergence being extreme and the eyeball slightly prominent. It can be rotated outward one centimetre, and there is slight rotary movement noticed when an attempt is made to turn the eye downward and outward. From the position of extreme divergence, the right eye can be rotated inward about two millimetres. The pupil is absolutely fixed, horizontally oval, its long diameter six millimetres, and its short four and one-half millimetres. The central color perception is good, and the form field normal.

Vision is $\frac{6}{60}$, or somewhat better, if the test card is held 1 Read before the American Ophthalmological Society, July, 1895. 
downward and to the right. The optic dise is gray-red in color, nearly round, and contains a small physiological cup. The scleral ring is sharply cut to the temporal side, and there is a slight remnant of the choroid ring on the nasal edge of the nerve-head. The general retinal circulation is normal.

The left eye presents no abnormality, vision and accommodation being natural and the ophthalmoscopic changes unimportant, possibly slight grayness in the deeper layers of the disc, with superficial capillarity. The iris movements are normal.

Briefly recapitulated, the points of this case are the following: Right oculo-motor palsy at the age of one and one-balf years; recovery in six weeks; frequent recurrence of the ocular palsy, associated with severe neuralgia and followed by complete recovery of the paralyzed muscles during the intervals, until the patient's fourth year, when the divergence remains, but the ptosis successively recurs and disappears as heretofore; finally, permanent ptosis at the twentyeighth year, after the most violent attack of pain of the whole series, and the present appearance of com. plete permanent right oculo-motor palsy. Other nerves are not involved in this case, for example, the abducens, facial, or trifacial.

It is unnecessary to review the literature of this affection, which has recently been done in a masterly manner by Dr. Philip Coombs Knapp. ${ }^{2}$ From Dr. Knapp's researches we learn that about forty cases of this affection have been reported; that it affects the two sexes nearly equally (twenty-one women, eighteen men); that in the majority of cases the disease begins in early life and usually, as in my case, with vomiting and severe pain, and that soon after the pain oculomotor palsy has occurred.

According to Knapp, after a varying interval, from a week to four years, the attack recurs, rarely at a definite period, for example, the menstrual epoch. If the clinical history of $m y$ patient may be trusted, in her early life the attacks recurred with greater frequency than the shorter intervals just mentioned, namely, bi.weekly.

In a certain number of the cases, although there was complete recovery from the ocular palsy, in the intervals between the earlier attacks, a gradually increasing paralysis was observed, which finally became permanent (Roosa's case and my own).

In three autopsies thus far reported (Knapp, loc. cit.) the following lesions were found: inflammatory thickening of the nerve (Gubler); tubercular mass pressing on the nerve (Weiss); fibrochondroma separating, without destroying the nerve fibres ('Thomson aud Richter). Knapp concludes "that recurrent oculo-motor palsy is due to some vascular change, intlammatory or edematous, in a focal lesion involving the root of the third nerve. As the edema or exudation subsides, the conducting power of the nerve is wholly or partially restored, and the paralysis disappears. In some cases the lesion may involve several nerves, and the exudation may affect only a part of the lesion, involving different nerves at different times. As the lesion progresses, it may finally affect the nerve so far as wholly to destroy its conducting power, leading, as in Roosa's case, to permanent and total paralysis." 'The last sentence describes accurately the course of events in the case reported to-day.

Treatment - mercury, iodides, strychuia - was

2 Boston Medical and Surgical Journal, vol. cxxxi, pp. 308-312, 1894. faithfully tried without avail; and Dr. Bochroch, Chief of the Clinic for Diseases of the Nervous System, carefully and skilfully used electricity for several months without favorably affecting the paralyzed levator.

\section{THE CONDITION OF THE NOSE IN PHTHISI-} CAL PATIEN'TS.

\author{
BY J. PAYSON CLARK, M,D.,
}

Physicianfor Diseases of the Throat, Massachusetts General Hospital.

IT seems not out of place as a preface to the following observations to review briefly our present knowledge of the functions of the nose as a respiratory organ, and of the structures most necessary to. the proper carrying on of these functions. Bosworth, writing on this subject, says: "The nasal passages" contain an exceedingly important, perhaps the most. important, and certainly the most intricate apparatus, connected with the function of respiration, of the whole respiratory tract, and one on whose normali functional activity depends the integrity of the whole: of the mucous membrane of the respiratory tract below."

Before speaking of this "intricate apparatus" let: us see what are the functions which it should normally perform. The two most important functions of the nasal chambers are to thoroughly warm and moisten the inspired air. Another function apparently less thoroughly performed is the arresting of mechanical dust, while a possible fourth, not as yet sufficiently established to rank with the others, is the destruction or diminution of the vitality of pathogenic germs. It is only comparatively recently that the existence of any of these functions has been recognized and demonstrated. In 1885 Bosworth, largely from clinical observation, advanced the theory that the five thousand grains of water, estimated by physiologists to be taken up in twenty-four hours by the inspiratory current of air from the whole respiratory apparatus, is derived solely from the nasal chambers. He further stated that " it is absolutely necessary and essential for the integrity of the lower air-passages that the air which reaches them should be so far charged with moisture that they should not be robbed of any of their secretion."

The organs for serous transudation are the large plexuses of blood-vessels covered by mucous membrane and lying on the inferior and middle turbinated bones, the so-called erectile tissue of the turbinated bodies. The special method of this transudation we do not yet know, unless it may be through the minute canals, running at right angles to the mucous membrane, penetrating to the lymph channels, as described by Chatellier. 'These large reservoirs of blood must also be, to a great extent, the source of the beat which the inspired air acquires in passing through the nose. Aschenbrandt proved in a series of experi. ments published in 1886 that the temperature of the air in passing through the nose is raised to $86^{\circ} \mathrm{F}$. As the temperature of the expiratory current in ordinary respiration is $86^{\circ} \mathrm{F}$., the warming of the air in respiration must be done exclusively by the nose. $\mathrm{He}$ also found that air in its passage through the nose is saturated with moisture, and that mechanical dust is completely arrested in the nose. Kayser's observations in the same direction confirmed those of Aschen- 